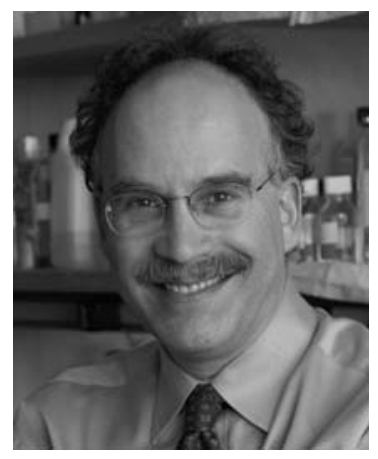

\title{
A Conversation with Daniel Haber
}

\author{
Interviewer: ElizABeth McKenNa \\ Associate Editor, Cancer Discovery
}

Daniel Haber is the Kurt J. Isselbacher Professor of Oncology at Harvard Medical School and Director of the Massachusetts General Hospital Cancer Center.

Elizabeth McKenna: Can you briefly introduce the topic of circulating tumor cells (CTCs)?

Dr. Haber: Circulating tumor cells are cancer cells that are found in the blood. This is how cancer spreads from a primary tumor in an organ to give rise to metastases. These cells are rare, and they're surrounded by many blood cells. We've spent a lot of time developing technologies to pull these cells out of the blood so we can study both metastasis, as well as cancer progression.

Some techniques use the fact that cancer cells are somewhat larger than blood cells so they try to filter them, but some cancer cells are not that large so you can miss a lot that way. Other technologies involve using antibodies against epitopes on tumor cells or epithelial cells to pull them out; those have become standard in the field. The challenge is that some epithelial cells become more mesenchymal and some cancer cells have different epitopes, so it's hard to get a reliable antibody to always pull out tumor cells.

The technologies that we've used in collaboration with Mehmet Toner, a bioengineer at Massachusetts General Hospital, purify out the normal cells. If you use an antibody against white blood cells to deplete them from a blood specimen and you remove the red blood cells and platelets, then you're left with the cells that don't belong in the blood: the tumor cells. That's hard to do; you need a technology that is really efficient at purifying away a large excess of normal blood cells. That's a big advantage in the field of microfluidics, where you can line up the cells in such a way that they pass single file through a channel. You can attach a magnetic particle with an antibody to the cells of interest and then pass them through a magnet so you can separate them. The technology has gotten more complex but also more powerful, to the point where you can reliably pull out these cells and ask the questions that you've always wanted to ask.
Elizabeth McKenna: Are CTCs similar to the parental tumor or have they diverged in any way? How are they generated and which ones are selected for metastasizing?

Dr. Haber: There's a lot of heterogeneity. Early cancers can shed cells into the blood way before any metastases occur. When you capture cells from a blood sample, maybe $90 \%$ or so are cells that are destined to die, but some of them could be alive and some of them could be endowed with the potential to give rise to metastasis. Recently, we've been able to culture some of them and the ones that grow in the laboratory look different. They grow in clusters, they grow under hypoxic conditions, and they're highly tumorigenic when you inject them back into mice. There's clearly a subpopulation in the blood that are bad actors.

Elizabeth McKenna: Can you learn anything about the development of the original tumor by collecting or culturing CTCs?

Dr. Haber: Technically, you can apply multiple assays to analyze CTCs at the single cell level. You can stain in situ for all kind of markers at the protein or RNA levels. You can pick individual cells and do RNA sequencing or do whole-genome amplification and look for mutations. The technologies have to be adapted for minute amounts of material, but those are available now and you can access the heterogeneity of CTCs. In a recent study looking at advanced prostate cancer, we could see how-even within an individual patient-different cells had different markers and different genetic abnormalities. It's a helpful way of looking at the complexity of cancer.

The most important thing from a clinical standpoint is that you can do this noninvasively with a blood test, and you can do this over and over again. Increasingly, we have these effective treatments for cancer and the more effective your treatment is the more the cancer shrinks, but when it grows back, it has different properties. You're

(C) 2016 Haber. This article is distributed under the terms of the Creative Commons Attribution-NonCommercial License, which permits reuse and redistribution, except for commercial purposes, provided that the original author and source are credited. 
not going to be successful at treating it a second time with the drug that you used the first time. Cancers evolve and the great potential of liquid biopsies is that you can sample tumors repeatedly to see how the cancers change in response to therapies.

Elizabeth McKenna: What needs to be addressed to demonstrate that liquid biopsying is going to have clinical utility?

Dr. Haber: There are three different technologies, all at different stages. You can look at whole CTCs, where you can do anything from culture them to look at protein, RNA, or mutational changes. There's free plasma DNA, which you can analyze primarily looking at mutations at the DNA level. There are also technologies to try to isolate exosomes: little particles of cellular material, including mostly RNA, but other things as well. Ultimately, there will be different applications for each one in the clinic. There are some things that you'll do with one and some with another, depending on whether a particular cancer has recurrent mutations or RNA changes or other markers that are clinically relevant.

The most obvious application is trying to figure out the mechanism of resistance when you give a treatment and the tumor shrinks but then comes back. There are now second-, third-, and fourth-generation treatments and you want to guide those based on the mechanism of resistance. If blood-based biopsies give you a sense of that mechanism, you'll know how to treat the patient. As those methods become more applied, I think they'll also be applied earlier in diagnosis. There are many times when the primary biopsy isn't so good or when it takes time to get a primary biopsy. You could see liquid biopsies moving earlier in the course of treatment.

Also, some of these cells in the blood have different properties, and that raises the question of different targets. There's a lot of interest in why tumor cells in the blood are more sensitive to different stressors such as oxygen stressors or loss of anchorage dependence. We might have new treatments for cancer based on suppressing their survival in the blood. That would be really exciting.

Elizabeth McKenna: Are tests for CTCs or cell-free DNA being incorporated into clinical trial design? What bar needs to be met for this to be introduced into clinical practice?

Dr. Haber: Both circulating tumor DNA as well as CTCs are involved in a large number of clinical trials. For them to gain acceptance and really become part of clinical care you have to have a demonstrated clinical benefit, but also there is a need for better reimbursement structures that will make them clinically viable. A challenge for the field is that as treatments have gotten better, the diagnostics become more important to guide these treatments. We need to put more effort in supporting diagnostics so that we can direct therapeutics more effectively.

Elizabeth McKenna: Is there a particular disease where these liquid biopsies might change practices in the near future?
Dr. Haber: One is where you have targeted therapies and they give rise to a couple defined mechanisms of resistance. If you have mutation A, you need this treatment; if you have amplification B, you need that treatment. It really makes a difference. I could see that happening relatively quickly in cancers such as lung and breast cancer, potentially melanoma. Also, there are cancers like prostate and breast cancer, where tumors spread to bone, which is challenging to biopsy. For these cancers, especially where some can be fairly indolent and others can be aggressive and where there are different medical therapies available, that's where blood sampling would be really helpful.

Elizabeth McKenna: Moving back to the biology of CTCs, what are the next big unanswered questions in the field?

Dr. Haber: We're seeing new pathways that are relevant to CTC biology (i.e., tumor cells that are transiently present within the bloodstream, without their usual surroundings, survival signals, and basement membrane attachment) that we hadn't appreciated before based on tumor analyses. The idea that some therapeutics could target CTCs is really intriguing. Personally, I also want to see CTC studies enabling us to diagnose cancer at an earlier stage. If you can diagnose cancer when, instead of a trillion cells, there are only a million cells, then many treatment options become so much more effective, since there are so many fewer cancer cells to harbor drug resistance. The challenge is that most early diagnosis of cancer is fraught with risk: You pick up noninvasive cancers; you have false positives. Blood-based diagnostics will have a major contribution here, because if a cancer cell invades the blood it's probably unlikely to be an indolent "well behaved" cancer cell. If we can combine bloodbased diagnostics with other tools that we now have for early detection of cancer, we could have a really big impact.

Elizabeth McKenna: You think that the presence of CTCs will occur before any change that would be detectable by imaging per se, so you could use it to track the disease before it might be detectable otherwise.

Dr. Haber: I think we will need a combination of assays. For example, in 20 patients with localized prostate cancer we could find CTCs in about a quarter to a fifth of them before surgery, and after surgery the CTCs were gone, and none of these patients have relapsed with 4 years followup. It's an example of how a subset of patients will have tumors that shed cells into the blood before metastases are established, and that offers a diagnostic opportunity. It could be the same in lung, in breast, or other types of cancers. Of course, it won't be true in every patient and we will have to combine blood-based assays with mammogram or a prostate-specific antigen (PSA) tests or lung CT scan screening. There's a lot to do to test and validate, but it's a whole new dimension of diagnostics.

Elizabeth McKenna: Are there ongoing clinical trials to see if the number of CTCs correlates with the response? 


\section{A CONVERSATION WITH DANIEL HABER}

Do you need large number of patients to make it a clinical benchmark?

Dr. Haber: That's of course the challenge. In a patient with cancer who's being treated, that patient is his or her own control in testing whether CTCs follow or predict clinical course. On the other hand, if you're looking at early detection, even in high-risk individuals, the number of patients or healthy individuals at-risk you need to study is considerably higher. You have to design those studies very carefully. For early detection applications, the test has to be even more reliable and robust. You can't make mistakes, including false positives. It's a whole new dimension to the CTC field, but ultimately it could have a tremendous impact on the way we monitor for cancer. 


\section{$\$_{\text {CSH\& }}^{\infty}$ Cold Spring Harbor Symposia SYMPOSIA On Quantitative Biology}

\section{A Conversation with Daniel Haber}

Cold Spring Harb Symp Quant Biol 2016 81: 311-313 originally published online January 5, 2017 Access the most recent version at doi:10.1101/sqb.2016.81.031419

Creative This article is distributed under the terms of the

Commons http://creativecommons.org/licenses/by-nc/4.0/, which permits reuse and

License redistribution, except for commercial purposes, provided that the original author and source are credited.

Email Alerting Receive free email alerts when new articles cite this article - sign up in Service the box at the top right corner of the article or click here. 